\title{
Guest editorial: Evolving autonomous systems under realistic environments
}

\author{
Seiichi Ozawa $\cdot$ Hisashi Handa
}

Published online: 10 November 2011

(C) Springer-Verlag 2011

In the conventional machine learning approaches, we sometimes have put somewhat unrealistic assumptions for training/test data such as sufficiency, ideal statistical property (e.g., Gaussian, i.i.d.), stationary process, complete observations (i.d., no missing variable), etc. These assumptions ensure the convergence and the universality of learning algorithms, the stability of system behaviors, and so on. However, considering our daily life, we can easily find the cases that violate such assumptions.

In face recognition, for example, since human faces have large variations by expressions, lighting conditions, makeup, hairstyles, and so forth, it is hard to consider all variations of face in advance. Thus, this makes quite difficult for a system to ensure the robustness over the spatial and temporal variations of human faces. Actually, conventional face recognition systems can achieve excellent performance when tested over a limited set of face images. However, it could drop rather drastically when they are operated in a practical environment. This is because the training set of face images may be either insufficient or inappropriate for future events. Even though a large amount of face images are collected when constructing a face recognition system, all the variations that will happen in future cannot be considered in advance. Therefore, a realistic solution for this is that a

\footnotetext{
S. Ozawa

Graduate School of Engineering, Kobe University,

Kobe, Japan

e-mail: ozawasei@kobe-u.ac.jp

H. Handa $(\bowtie)$

Graduate School of Natural Science and Technology,

Okayama University, Okayama, Japan

e-mail: handa@sdc.it.okayama-u.ac.jp
}

system is learned incrementally whenever it misclassifies objects. Consequently, it is a natural assumption under realistic environments that face images for training are given as a stream.

Here is another example of face recognition that we should consider under a realistic situation. In general, the objects we encounter in our daily life can be described in various ways. For example, from a human face, we recognize multiple features such as name, gender, age, health condition, etc. Thus, when we see a person, we might have to answer the name of the person from his/her face. In some cases, however, we might have to answer the age, gender or health condition. The right answer depends on a context we are facing. Generally, it is believed that we humans learn and perform multiple pattern recognition tasks in parallel or sequentially. In machine learning, the recognition of a feature (e.g., name, gender, age, etc.) is usually defined as an individual task; that is, the recognition task to answer the name of a person is called person identification, the recognition tasks to answer the gender and the age are called gender recognition and age estimation, respectively. From the above facts, it is natural for us to expect that a learning system can also learn multiple tasks like humans (Thrun et al. 1998).

There are many situations other than the above that we should consider in constructing a learning systems under realistic environments. For example, a part of training data might have no supervised information (e.g., class labels and target signals); therefore, a learner should predict the information to generate training data on its own. In some case, data distributions could be changed over time with slow or sudden drifts. And there might be no prior information on useful features of high-dimensional inputs; then, a learner should select or extract optimal features dynamically. Furthermore, training data could also include 
outlier, missing values, and some other uncertainty. To deal with training data under such tough conditions, conventional static models are no longer useful. A learning system should be evolvable and/or adaptive to find optimal solutions of a given task with some autonomy (Kasabov 2007; Angelov et al. 2010).

The mission of this special issue is to introduce the latest technologies in machine learning that tackle realistic environments. The meaning of 'Evolving' in the title of this special issue is not only restricted to so-called "Evolutionary Process" but also includes any kinds of "Autonomous Learning Processes" that adapt to and/or earn the useful knowledge from realistic environments. To collect excellent papers, we organized a special session with the same title in the Pacific Rim International Conference on Artificial Intelligence (PRICAI) 2010 held in Taegu, Korea (30th August through 2nd September, 2010), which is the bi-annual international conference on a broad sense of artificial intelligence including neural networks, machine learning, and data mining. For our call, 11 papers were originally submitted to the organized special session, and finally 8 papers were accepted as full papers. Considering that the acceptance rate of PRICAI2010 is around $30 \%$, the quality of the papers submitted to this special session is quite high and actually we had great discussions in the special session. After the conference, we immediately called for the submissions to the special issue of Evolving Systems. Until the end of 2010, we received five submissions, and finally four papers are accepted as regular papers. Actually, they cover a large part of the problem domains that are supposed to be in this special issue. However, to be more complete for our mission, following the suggestion by the Editor-in-Chief of Evolving Systems, Dr. Angelov Plamen, we have willingly decided to invite another excellent paper as the fifth paper. The followings are brief summaries of the accepted papers in this special issue.

The paper, "Adaptive Bidding in Combinatorial Auctions in Dynamic Markets," by Xin Sui and Ho-fung Leung, proposes a new adaptive bidding strategy for combinatorial auction-based resource allocation problem in a class of dynamic markets. In such realistic markets, a good bidding strategy is not obtained either by analytical methods and conventional machine learning approaches since the market information cannot be assumed to be known in advance and the market situations are always changing. For this challenging problem, the proposed method introduces a bidding strategy based on a scenario in which the first-price sealed-bid combinatorial auctions are used to allocate computational resources among a group of users, each of whom needs a bundle of resource to perform their own task. The authors show that the performance of the adaptive strategy is much better than that of the random strategy, and is very good when compared with an oracle strategy.

The paper, "On the Effect of Dimensionality Reduction by Manifold Learning for Evolutionary Learning," by Hisashi Handa, proposes the use of Manifold Learning for Evolutionary Learning with redundant sensory inputs in order to avoid the difficulty of designing the allocation of sensors. The proposed method consists of two stages: The first stage is to generate a mapping from higher-dimensional sensory inputs to a lower dimensional space, by using Manifold Learning. The second stage is to use Evolutionary Learning for learning a control scheme. To implement the basic idea, the author adopts Isomap and LLE as the manifold learning algorithms and the instancebased policy optimization as the evolutionary learner. In addition, a metric of relative error of distances between original input space and reduced space is introduced. The author applies the proposed scheme to robot navigation problems, where robots with a large number of range sensors are required to learn their optimal policy quickly through limited experiences.

The paper, "Incremental Model Selection and Ensemble Prediction under Virtual Concept Drifting Environments," by Koichiro Yamauchi, proposes an incremental model selection method under a sort of dynamic environments. Under the virtual concept drifting environments, the distribution of the observed samples is considerably different from the one of the cumulative dataset. In order to cope with such dynamic environments, this paper introduces the weighted objective function and the model-selection criterion based upon the predictive input density of the learning samples. Moreover, this paper proposes the prediction of the input density by using an ensemble radial basis function neural network method.

In the paper, "A real-time personal authentication system based on incremental feature extraction and classification of audiovisual information," by Young-Ming Jang, Minho Lee, and Seiichi Ozawa, an effective and practical personal authentication system based on face and voice is introduced. An incremental principal component analysis extracts features and updates them incrementally. The paper shows that the proposed system can enhance the test performance incrementally without serious forgetting, and that the multi-modal feature effectively increases the robustness of the personal authentication system in noisy environments.

Acknowledgments The guest editors would like to thank the authors who responded to the call and all of the anonymous reviewers who gave useful comments to the authors and keep the tight review schedule. The guest editors would like to thank the Editor-in-Chief, Dr. Angelov Plamen, for his highly professional attitude and dedication to the quality of the journal and for giving the opportunity to have such a timely special issue. 


\section{References}

Angelov PP, Filev DP, Kasabov N (eds) (2010) Evolving intelligent systems: methodology and applications. Wiley-IEEE Press, New York
Kasabov N (2007) Evolving connectionist systems: the knowledge engineering approach. Springer, London

Thrun S, Pratt L (1998) Learning to learn. Kluwer Academic Publishers, Dordrecht 